\title{
Imaging ecografico in dialisi peritoneale
}

\author{
Vincenzo Barbera ${ }^{1}$, Luca Di Lullo ${ }^{1}$, Marco Federici ${ }^{2}$, Giovanni Otranto ${ }^{1}$, Francesca Santoboni ${ }^{1}$, Alberto Santoboni ${ }^{1}$ \\ ${ }^{1}$ UOC Nefrologia e Dialisi, PO L. Parodi Delfino, ASL ROMA 5, Colleferro (RM) \\ ${ }^{2}$ UO Diagnostica per Immagini, PO L. Parodi Delfino, ASL ROMA 5, Colleferro (RM)
}

\begin{abstract}
Sonography in peritoneal dialysis patients
Traditionally computed tomography (CT) and more recently magnetic resonance (MR) are the imaging modalities of choice for the evaluation of peritoneum and peritoneal cavity in primary and secondary pathologic conditions. Nevertheless, ultrasound (US) also may be a valuable, accurate, safe, relatively inexpensive and readily accessible diagnostic tool to investigate the diseases that can affect the peritoneal membrane. US is able to provide quantitative information demonstrating much smaller quantities of free intraperitoneal fluid (ascites) being more sensitive than $\mathrm{CT}$ in this regard. Sonographic characteristics of ascites allow to detect the presence of blood, pus or neoplastic cells in echogenic fluid. Sonography is very helpful when planning and performing interventional procedures on abdominal wall such as: fine-needle aspiration, core biopsy and drainage: with modern devices lesions of less than $4 \mathrm{~mm}$ may be easily targeted. Ultrasonography is used in the assessment of several procedures and complications in peritoneal dialysis (PD) patients. Some of them are well documented whereas other indications are less clear. Peritoneal ultrasound fields of applications include some of the following: correct patients' enrollement, ultrasound-guided catheter's placement, potential diagnosis of exit site and tunnel infections and/ or peritonitis (both infectious and/or sclerosing) and leakage amount's assessment. The purpose of this review is to describe an US technique for optimal detection and localization of the main complications commonly encountered in patients affected by chronic kidney disease undergoing peritoneal dialysis.
\end{abstract}

Keywords: Exit site infections, Peritoneal dialysis, Sonography, Tunnel infections, Visceral slide

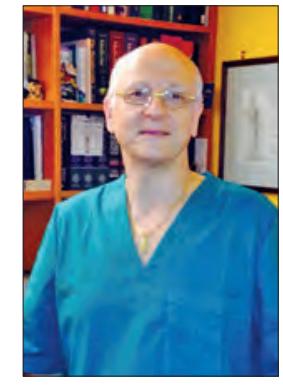

Vincenzo Barbera

\section{Introduzione}

La tomografia computerizzata (CT), tradizionalmente, e la risonanza magnetica (MR), più di recente, rappresentano le principali tecniche di imaging per la valutazione radiografica del peritoneo e della cavità peritoneale. Tuttavia, anche l'ecografia si è rivelata una metodica estremamente accurata, prontamente disponibile nonché sicura ed economica per lo studio della sierosa peritoneale, consentendone una corretta valutazione dell'anatomia e delle lesioni eventualmente presenti. Utilizzando sonde adeguate è possibile visualizzare la presenza di aderenze nonché rica-

Accepted: September 18, 2016

Published online: November 21, 2016

Indirizzo per la corrispondenza:

Dr. Vincenzo Barbera

UOC Nefrologia e Dialisi

PO L. Parodi Delfino (ASL ROMA 5)

Piazzale Aldo Moro 1

00031 Colleferro (RM)

docvic@tiscali.it vare informazioni di carattere quantitativo, essendo l'ecografia, a tal riguardo, assai più sensibile della CT. È, così, possibile rilevare la presenza di modeste quantità di fluido libero intraperitoneale, permettendo una corretta definizione dell'ascite ed evidenziando l'eventuale presenza di sangue, pus o cellule neoplastiche in un versamento particolato. La metodica ecografica si rivela, poi, particolarmente utile nell'esecuzione di procedure interventistiche sulla parete addominale, come: agoaspirato, biopsie e posizionamento di drenaggi. I moderni dispositivi consentono il prelievo da lesioni aventi dimensioni inferiori ai 4 millimetri.

Accanto a tali indicazioni di carattere più generale, l'ecografia ben si presta alla valutazione del peritoneo nel paziente sottoposto a trattamento con dialisi peritoneale sia per quello che riguarda lo studio pre-impianto e il posizionamento del catetere di Tenckhoff che per quello che riguarda le principali complicanze connesse alla metodica, come: le infezioni dell'exit-site e del tunnel sottocutaneo, le peritoniti, il leakage di fluido peritoneale e la diagnosi di peritonite sclerosante e incapsulante.

Malgrado ciò, la letteratura scientifica è assai avara di dati sulle tecniche di scanning ecografico del peritoneo, soprattutto se confrontata con quella assai più vasta riguardante gli organi solidi addominali. Le ragioni di tale mancanza sono da ricercarsi soprattutto in un training inadeguato e nella scarsa familiarità con i comuni aspetti ecografici riscontrabili in corso 
di patologia peritoneale. Né, d'altra parte, possono trascurarsi i limiti fisici degli ultrasuoni (US) conseguenti alla presenza di gas intestinali che, ostacolando l'intera visualizzazione della cavità peritoneale, rendono difficile le corrette esecuzione e interpretazione dell'esame.

Scopo della presente review è, pertanto, quello di descrivere la tecnica US per un corretto approccio all'identificazione e alla localizzazione delle patologie peritoneali più frequenti in corso di dialisi peritoneale (PD), illustrandone gli aspetti ecografici più caratteristici e clinicamente utili per il nefrologo.

\section{Anatomia ecografica della parete addominale}

L'ecografia rappresenta una metodica fondamentale nella valutazione della parete addominale e delle sottostanti strutture. Le basi del successo diagnostico poggiano sulla completa conoscenza delle strutture indagate; si ritiene, pertanto, opportuna la dettagliata descrizione della tecnica US, in grado di consentire il corretto riconoscimento degli elementi anatomici incontrati e l'ottimale interpretazione dei reperti patologici eventualmente presenti.

Inizialmente, una panoramica del peritoneo e della cavità peritoneale può essere ottenuta mediante una sonda convex di frequenza standard (3.5-5.0 MHz), il che permette la valutazione completa del contenuto addominale e pelvico, ivi inclusi i visceri solidi. L’immagine così ottenuta può essere regolata in maniera tale da includervi la profondità della cavità addominale o pelvica: tale regolazione consente di fornire una buona prospettiva all'immagine stessa. Successivamente, utilizzando sonde lineari, anche ad alta risoluzione (12-15 MHz o a frequenza più elevata), è possibile valutare meglio la struttura ecografica del peritoneo ed eventualmente definire eventuali lesioni riscontrate nel campo vicino. Nei soggetti di sesso femminile, se necessario, è possibile utilizzare la sonda endocavitaria per lo studio del peritoneo pelvico, specialmente nel sospetto di carcinomatosi peritoneale o di processi flogistici acuti (soprattutto PID, Pelvic Inflammatory Diseases). Oltre alla valutazione standard dell'utero e delle ovaie, è opportuno completare l'indagine con lo studio delle pareti laterali della pelvi e del cavo di Douglas per una corretta definizione del peritoneo parietale.

Nello spessore della parete addominale sono riconoscibili ecograficamente tre differenti strati che, procedendo dalla superficie, sono così rappresentati: 1) strato superficiale, composto da cute e tessuto adiposo sottocutaneo; 2) strato intermedio, formato dai muscoli che rivestono la cavità addominale; 3) strato profondo, contenente il grasso pre-peritoneale e il peritoneo parietale. Lo spessore del grasso sottocutaneo varia considerevolmente a seconda degli individui. I lobuli adiposi appaiono come noduli ipoecogeni, di forma rotondeggiante od ovalare separati da setti iperecogeni. I vasi sanguigni, penetrando al loro interno, possono interromperne l'armoniosa struttura e il regolare echopattern: nei casi dubbi, l'accensione del box colore consente la loro differenziazione.
Lo strato muscolare della parete addominale mostra un pattern simmetrico da ciascun lato della linea alba, dal cui confronto è possibile ottenere informazioni utili. In una scansione assiale, i ventri muscolari del retto addominale appaiono come strutture ovoidali ipoecogene con fini echi interni. Lateralmente ad essi, appaiono chiaramente visibili i tre strati formati dai muscoli obliquo esterno, obliquo interno e trasverso dell'addome.

Il terzo strato o strato profondo della parete addominale è solitamente quello più sottile; qui lo spessore del grasso preperitoneale misura solo pochi millimetri. Posteriormente ai due ventri del muscolo retto, a livello della linea mediana e in corrispondenza del terzo superiore è solitamente presente un maggior accumulo di tessuto adiposo. Quest'area può agire come una lente acustica, dando origine al ben noto «artefatto di sdoppiamento dell'immagine» (1). Nel grasso preperitoneale sono ben visibili i vasi epigastrici: il loro corretto riconoscimento è essenziale prima di intraprendere qualsiasi procedura interventistica sulla parete addominale o attraverso di essa al fine di minimizzare il rischio emorragico.

\section{Anatomia ecografica del peritoneo e dei recessi peri- toneali}

Il peritoneo è una membrana sierosa delimitata da un singolo strato di cellule epiteliali appiattite che si riuniscono a formare il mesotelio. Può essere suddiviso in un foglietto parietale e in un foglietto viscerale. I due foglietti delimitano uno spazio virtuale, la cavità peritoneale, contenente al suo interno alcuni millilitri di un fluido citrino, il liquido peritoneale. In condizioni normali, il peritoneo non è visualizzabile ecograficamente a causa della sua estrema sottigliezza e dell'intimo contatto con le strutture che involge. Solo occasionalmente, e in determinati distretti, si rende evidente sotto forma di una delicata linea iperecogena, di spessore uniforme. Ciò è reso possibile poiché la sierosa viene a essere compresa tra due strati di tessuto adiposo che, con la propria peculiare ecogenicità, costituiscono un enhanced naturale (2). Analogamente, in condizione di normalità, non è possibile dimostrare all'US la cavità peritoneale poiché, come accennato in precedenza, quest'ultima rappresenta solo uno spazio virtuale.

Le anse intestinali sono visualizzabili soprattutto distalmente alla sierosa peritoneale; sono dotate di movimenti autonomi, asincroni rispetto agli atti del respiro. La presenza di ascite magnifica notevolmente la rappresentazione ecografica del peritoneo mettendo, nello stesso tempo, in evidenza la presenza di eventuali lesioni.

Il mesentere è un'ampia piegatura del peritoneo, a forma di ventaglio, che si estende obliquamente dalla seconda vertebra lombare alla fossa iliaca destra, congiungendo il digiuno e l'ileo alla parete posteriore della cavità addominale. Nel suo spessore sono altresì contenuti vasi sanguigni e linfatici, nervi, linfonodi e una percentuale variabile di tessuto adiposo. Derchi et al (3) descrivono ecograficamente le pieghe mesenteriali come una serie di strutture allungate, di spes- 
sore variabile dai 7 ai 12 millimetri, separate tra loro da echi speculari corrispondenti alle superfici peritoneali. Contrariamente all'intestino, il mesentere non è dotato di movimenti peristaltici; le strutture anecogene evidenti al suo interno ne rappresentano i vasi sanguigni. II mesentere può visualizzarsi ecograficamente conducendo una scansione obliqua in corrispondenza del quadrante addominale inferiore sinistro, approssimativamente parallela ai vasi iliaci; esso appare più evidente nei pazienti obesi. In presenza di un versamento ascitico, il mesentere è facilmente riconoscibile all'US sotto forma di una serie di digitazioni liberamente flottanti separate da liquido libero. La presenza di eventuali aderenze può essere rivelata dalla fusione dei foglietti e dalla perdita di fluttuazioni del mesentere all'interno del fluido.

I legamenti e i mesi che si estendono tra il peritoneo e i diversi organi addominali suddividono la cavità peritoneale in diversi spazi e subspazi, la cui comprensione è indispensabile per un corretto inquadramento diagnostico delle patologie di tale sierosa. II mesocolon trasverso divide il peritoneo in due compartimenti: lo spazio sovramesocolico, compreso tra il diaframma e il mesocolon trasverso, e lo spazio sottomesocolico, contenente le anse intestinali e che si estende dal mesocolon trasverso fino al piccolo bacino (4). Un terzo compartimento, delimitato da una linea virtuale passante per il carrefour aortico e il peritoneo che riveste il retto e la vescica, individua lo spazio pelvico o paravescicale, contenente il sigma e, nella donna, l'utero e gli annessi (5).

Il recesso epatorenale o tasca di Morrison rappresenta la suddivisione posteriore dello spazio sottoepatico, in rapporto diretto con il foglietto parietale posteriore, il quale dalla parete addominale posteriore si porta a rivestire la superficie anteriore del rene destro. Tale recesso costituisce, insieme alla pelvi, il punto più declive della cavità peritoneale a paziente supino, rappresentando, in tale decubito, la sede più frequente di raccolta di un eventuale versamento libero.

\section{Impiego degli ultrasuoni in dialisi peritoneale}

Anche se abbastanza comune, l'impiego degli US nelle procedure e nelle complicanze della PD presenta alcuni punti deboli che, in definitiva, sono quelli propri della metodica, primo fra tutti la stretta dipendenza dall'operatore. A ciò si aggiunge il progressivo perfezionamento delle tecniche di imaging radiografico e scintigrafico, che consente di ottenere informazioni diagnostiche sempre più precise e, talora, insostituibili (topografia del catetere peritoneale, distribuzione del liquido nella cavità peritoneale, leakage di parete e comunicazioni anomale: peritoneo-pleurica, peritoneo-vaginale). Ne consegue che, in PD, l'ecografia è utilizzata prevalentemente dal nefrologo piuttosto che dal radiologo, con il risultato di una scarsa messe di letteratura sull'argomento. Tuttavia, le opportunità offerte dagli US sono innumerevoli. Accanto alla rapidità di una prima diagnosi non invasiva, la metodica offre all'operatore esperto grandi sensibilità e definizione del dettaglio anatomico, ren- dendo spesso superflui ulteriori accertamenti radiologici, contrastografici e con radionuclidi, potenzialmente dannosi per il paziente e per la funzione renale residua.

Analizzeremo, pertanto, gli aspetti prevalentemente nefrologici dell'utilizzo degli US in PD con particolare riguardo allo screening del paziente candidato al trattamento con PD, al posizionamento del catetere di Tenckhoff e alle eventuali complicanze post-impianto, alle infezioni dell'exit-site e del tunnel sottocutaneo, nonché alla temibile peritonite sclerosante incapsulante.

\section{Screening ecografico del paziente candidato alla dialisi peritoneale}

L'idoneità del paziente candidato al trattamento con PD prevede una valutazione clinica che consideri, tra l'altro, l'eventuale presenza di estese aderenze addominali, malattie infiammatorie e/o ischemiche dell'intestino e diverticolosi del colon. La presenza di tali situazioni potrebbe, infatti, determinare un'insufficiente funzione dializzante della membrana peritoneale, sia direttamente (nel caso di estese aderenze) che indirettamente (nel caso di diverticoliti e malattie infiammatorie croniche intestinali). In condizioni normali, il peritoneo svolge una funzione protettiva sul contenuto della cavità addominale, consentendo lo scambio di molecole e la produzione di fluido peritoneale e realizzando, in tal modo, un ambiente in cui gli organi addominali siano in grado di funzionare adeguatamente. In caso di insulti di natura traumatica (chirurgici) o conseguenti a processi flogistici, viene attivata una serie di risposte biochimiche e cellulari aventi come obiettivo la riparazione delle zone di mesotelio danneggiate. Tali reazioni determinano la migrazione in situ di cellule infiammatorie nonché l'attivazione delle cellule mesoteliali residenti con produzione finale di un essudato fibrinoso. In funzione della gravità del danno subito, questo essudato può avere carattere transitorio (risolvendosi per fibrinolisi) o divenire più denso, come conseguenza del persistere della popolazione fibroblastica, dando, così, origine a fenomeni adesivi (le cosiddette «aderenze»).

Un'attenta e accurata raccolta anamnestica è già, di per sé, in grado di svelare alcune di queste condizioni; tuttavia, l'esecuzione di un esame ecografico mirato rappresenta un valido ausilio capace di confermare un sospetto clinico o di risolvere un dubbio diagnostico. La valutazione US dell'addome è routinariamente eseguita nello screening del candidato alla PD al fine di individuare eventuali controindicazioni o comorbidità da emendare (masse neoplastiche, visceromegalie, versamenti endocavitari, cisti, nefropatie ostruttive, litiasiche o infettive, colecistopatie, aneurismi dell'aorta addominale). Le basi anatomo-funzionali della valutazione US di eventuali aderenze si basano sull'osservazione che, in condizioni anatomiche normali, nello spazio peritoneale sono possibili solo determinati movimenti degli organi endoaddominali. In tale situazione, i visceri possono muoversi sia reciprocamente tra di loro che rispetto alla parete anteriore dell'addome. Di tali 
movimenti, chiamati «visceral slide», il più comune è quello longitudinale, movimento avanti-indietro dei visceri determinato dalle escursioni respiratorie del diaframma. Altre forze, sia interne (p. es., peristalsi) che esterne (p. es., manovre di ballottamento manuale dell'addome) sarebbero, a loro volta, in grado di causare movimenti degli organi addominali. La presenza di aderenze secondarie a precedenti chirurgici o ad altre condizioni cliniche determina una limitazione dei movimenti descritti. Siegel et al (6) introdussero per primi, nel 1991, la definizione di "visceral slide» descrivendo, altresì, una tecnica ecografica (vedi oltre) per la valutazione del movimento di «sliding». L'intento degli Autori era di realizzare una metodica di imaging non invasiva che consentisse loro di identificare e mappare eventuali aderenze prima delle procedure laparoscopiche. In tal modo, essi potevano disporre di una guida per il posizionamento del primo trocar (generalmente "blind») in aree prive di aderenze e, contemporaneamente, pianificare l'approccio alla regione d'interesse in maniera più rapida e sicura.

II rilievo US di aderenze si basa sulla presenza (o meno) nelle scansioni ecografiche di movimenti dei visceri addominali rispetto alla sovrastante parete anteriore dell'addome. L'esistenza di aderenze determina, infatti, una limitazione di tali movimenti svelabile ecograficamente come una restrizione del "visceral slide». La tecnica d'esame più frequentemente utilizzata consiste nell'effettuare, con il paziente in decubito supino, delle scansioni sul piano longitudinale mediante una sonda lineare di frequenza variabile tra i 5 e i 7.5 $\mathrm{MHz}$, la quale consente di ottenere il miglior campo di vista delle strutture situate nell'area di contatto con il trasduttore. In condizioni di normalità, le escursioni diaframmatiche permettono di evidenziare uno «sliding» viscerale spontaneo variabile tra i 2 e i 5 centimetri. Mediante le manovre di ballottamento manuale, effettuabili sia sul piano di scansione longitudinale che su quello trasversale, è, invece, possibile indurre uno scorrimento superiore a 1 centimetro. Definiremo, pertanto, come "ridotta» un'escursione viscerale inferiore al centimetro, sia spontanea che indotta. La ricerca e l'eventuale "mapping» delle aree di ridotti "viscera slides» rappresentano importanti elementi sia per il chirurgo che per il nefrologo. Tale conoscenza consente, infatti, al primo di scegliere il punto d'ingresso più adatto a un'eventuale chirurgia laparoscopica (o altra) e al secondo di valutare l'esistenza di regioni più o meno estese di peritoneo potenzialmente non utilizzabili come membrana dializzante.

In questi ultimi anni, l'ecografia del tratto gastrointestinale ha assunto un'importanza progressivamente crescente nel percorso diagnostico e terapeutico della patologia dell'apparato digerente, sia acuta che cronica. L'iniziale convincimento che gli US fossero uno strumento di imaging inappropriato nella valutazione dell'intestino trovava fondamento soprattutto nel fatto che le anse intestinali contengono quantità variabili di gas che, come noto, rappresentano il principale ostacolo alla propagazione degli ultrasuoni, determinando coni d'ombra e artefatti da riverberazione. A ciò si aggiungevano le difficoltà rappresentate dalla lunghezza e dalla tortuosità del tratto gastroenterico nonché dalla scarsa penetrazione della metodica US nei pazienti obesi. Tuttavia, nel tempo, il perfezionamento tecnologico nonché lo sviluppo di metodiche quali l'idrosonografia e l'ecocontrasto (CEUS, Contrast Enhanced U/traSonography) hanno fatto sì che l'ecografia divenisse uno strumento prezioso nella visualizzazione dell'apparato gastrointestinale. Gli scopi del presente lavoro consentono, tuttavia, I'analisi dei soli aspetti relativi alla tecnica $B$-mode nella diagnostica delle malattie croniche intestinali in generale e del morbo di Crohn in particolare, dovendo fare riferimento, come già detto, alle eventuali indicazioni all'inserimento o meno in un programma di trattamento con PD.

L'ecografia convenzionale del tratto gastroenterico viene eseguita con il paziente in decubito supino e non richiede alcuna preparazione specifica. Il digiuno protratto da almeno sei ore può, tuttavia, rivelarsi utile nel ridurre la quantità di fluidi e aria presenti nel lume intestinale e nel rallentare la peristalsi. La riduzione del contenuto gassoso può essere ottenuta facendo assumere liquidi al paziente per via orale oppure attraverso un sondino nasogastrico; in tal modo, è possibile anche separare le pareti intestinali e differenziare le anse del piccolo intestino tra loro. Cosi facendo, si rende più facilmente visualizzabile anche la parete posteriore (mesenterica) dell'intestino, spesso nascosta dietro sacche d'aria (7); quest'ultima può essere evidenziabile ancora meglio spostando i gas intraluminali con una compressione graduata dell'intestino.

Dal punto di vista tecnico, si raccomanda di iniziare sempre la valutazione con un'ecografia standard dell'addome utilizzando una sonda convex da 3-5 $\mathrm{MHz}$ e, successivamente, un trasduttore lineare ad alta frequenza $(5-10 \mathrm{MHz})$; ciò consentirà di ottenere una dettagliata risoluzione della parete del piccolo intestino. È possibile, così, riconoscere una struttura pluristratificata composta essenzialmente da cinque piani che, procedendo dal lume verso la mucosa, possono essere così distinti (Fig. 1): 1) iperecogeno: gas intraluminale; 2) ipoecogeno: mucosa; 3) iperecogeno: sottomucosa; 4) ipoecogeno: tonaca muscolare propria; 5) iperecogeno: sierosa. La stratificazione parietale e lo spessore murale rappresentano gli elementi più importanti in grado di rivelare la presenza di condizioni morbose: è opportuno, pertanto, che questi vengano misurati procedendo dal lume verso la sierosa. In condizioni normali, tale valore non supera i 3-6 millimetri, variando con la sede, l'età del paziente, lo stato di contrazione della muscolaris mucosae e la dilatazione dell'intestino. In letteratura, il valore di cut-off più frequentemente riportato per lo spessore parietale del piccolo intestino varia fra i 3 e i 4 millimetri (8). È opportuno, inoltre, valutare il grado di distensione delle anse intestinali che, in caso di ostruzione o malassorbimento, possono presentare un certo grado di dilatazione (diametro $>25$ millimetri) con un aumento del contenuto fluido al loro interno. La vascolarizzazione parietale e la peristalsi intestinale possono essere indagate mediante color- e power-Doppler o mediante CEUS. 


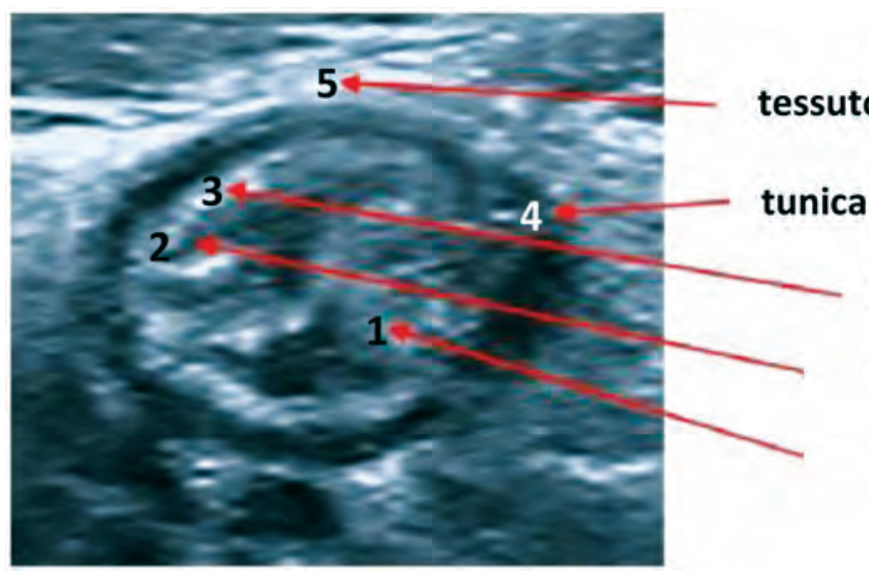

essuto adiposo/mesentere

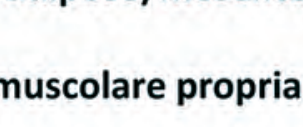

sottomucosa

mucosa

lume

Fig. 1 - Aspetto ecografico della parete intestinale (ileo): scansione assiale. Sono riconoscibili i 5 strati che compongono lo spessore parietale (v. testo): 1) lume: anecogeno; 2) mucosa: ipoecogena; 3) sottomucosa: iperecogena; 4) tunica muscolare propria: ipoecogena; 5) tessuto adiposo/mesentere: iperecogeno.

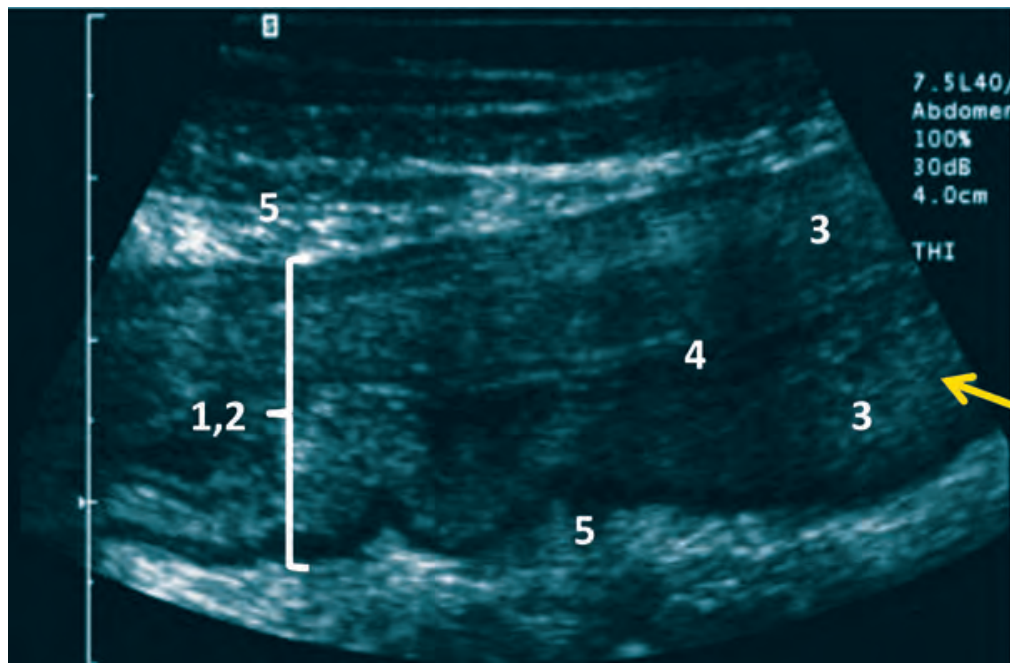

Fig. 2 - Caratteri ecografici del coIon in corso di morbo di Crohn. Si rilevano alcuni aspetti caratteristici: 1) ispessimento parietale $(>10 \mathrm{~mm})$; 2) perdita parziale della caratteristica struttura a strati della parete; 3) ispessimento dello strato sottomucoso; 4) riduzione del lume; 5) ipertrofia del tessuto adiposo mesenteriale.

I dati riguardanti l'accuratezza dell'imaging ecografico in corso di malattia di Crohn (CD) sono molto eterogenei; tuttavia, dalla metanalisi di Fraquelli et al emergerebbero una sensibilità variabile tra il 75 e il $94 \%$ e una specificità del $67-100 \%$ (8). Com'è noto, la malattia di Crohn può colpire l'intero tratto gastroenterico, sebbene la principale localizzazione sia generalmente rappresentata dall'ileo terminale. II piccolo intestino è coinvolto nel 30-40\% dei pazienti (con interessamento dell'ileo terminale nel $90 \%$ di essi), mentre, nel $40-55 \%$ dei casi, può esserci il contemporaneo coinvolgimento ileo-colico. Solo una piccola percentuale di casi, variabile dal $15 \%$ al $25 \%$ mostra un esclusivo interessamento del grosso intestino.

I criteri ecografici più comunemente utilizzati nella diagnosi della CD sono rappresentati dall'ispessimento della parete del piccolo intestino (con mantenimento o perdita della sua normale stratificazione) e dall'aumento della sua vascolarizzazione. Si parla di ispessimento parietale quando il diametro murale supera i 3 millimetri (Fig. 2). Tale valore permette di ottenere una sensibilità e una specificità diagnostiche rispettivamente dell' $88 \%$ e del 93\%. Qualora, invece, si utilizzino, come limite superiore, $4 \mathrm{~mm}$ la sensibilità si riduce significativamente (75\%), mentre si ha un aumento della specificità (97\%) (8). Tali parametri ecografici, avendo dimostrato un'eccellente riproducibilità, sono stati standardizzati nella diagnostica US della CD. Ciò vale soprattutto per quanto riguarda lo spessore parietale. II color- o il power-Doppler imaging utilizzano uno score semiquantitativo $(0=$ nessun segnale vascolare; 1 = spot colore appena visibili; 2 = discreta vascolarizzazione; 3 = marcata vascolarizzazione) per definire il grado di vascolarizzazione dell'ispessimento murale, rivelatosi particolarmente utile nella distinzione tra fase di attività e di remissione della malattia. Tale score mostra una buona correlazione soprattutto con lo score endoscopico di attività in adulti $(9,10)$ e bambini $(11)$. Al momento, non vi è alcuna scala obiettiva utile per determinare il grado di attività di malattia al Doppler US. 

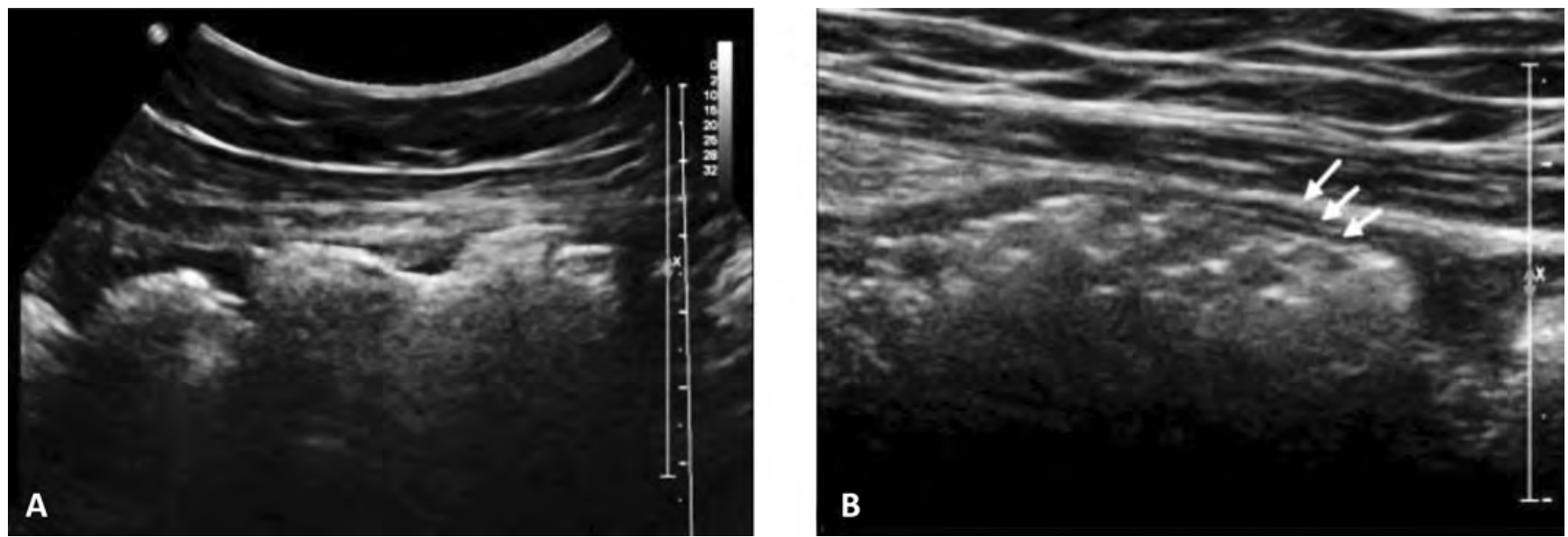

Fig. 3 - Normale aspetto ecografico del colon discendente (scansione longitudinale). (A) è evidente la tipica austratura del colon; (B) struttura laminare della parete del grosso intestino (frecce).

In aggiunta ai criteri descritti, la valutazione US delle strutture adiacenti peri-intestinali sembra in grado di fornire utili indicazioni circa lo stato reattivo della CD. L'ipertrofia del tessuto adiposo mesenteriale è correlata, infatti, con le fasi di attività clinica e con i parametri biochimici della $C D$, oltre che con la presenza di fistole e con l'ispessimento parietale. L'US si rivela, inoltre, assai sensibile nell'evidenziare la presenza di fluido libero nella CD (12). Anche l'esistenza di linfonodi locoregionali ha dimostrato una debole correlazione con le fasi di attività di malattia. Tuttavia, tale reperto non sembra essere specifico ed è, pertanto, necessario differenziarlo da altre malattie infiammatorie dell'intestino. Infine, I'US è particolarmente utile anche nella valutazione di altri aspetti patologici della $C D$, quali stenosi, fistole e ascessi.

La malattia diverticolare del colon (CDD) interessa circa il 5-10\% della popolazione di età superiore ai 45 anni e oltre I'80\% degli ultraottuagenari. Le dimensioni dei diverticoli possono variare da pochi millimetri a diversi centimetri e, solitamente, compaiono in corrispondenza del punto in cui i vasi sanguigni perforano la parete colica attraversandola. Sebbene possano essere presenti per l'intera lunghezza del colon, il sigma ne rappresenta la sede più frequente. Una volta comparsi, i diverticoli tendono a riempirsi di feci e ciò può condurre a una loro infiammazione, anche se la maggioranza degli individui tende a rimanere asintomatica per lunghi periodi. L'ostruzione del colletto diverticolare ad opera di feci e coproliti o l'infiammazione dello spazio pericolico ad opera di materiale alimentare possono condurre a una malattia acuta diverticolare $(A C D)$, meglio definibile come peridiverticolite, che può causare una perforazione con conseguente ascesso pericolico. L'imaging riveste, pertanto, un ruolo fondamentale nella diagnosi e nello staging dell'ACD. La CT permette la visualizzazione dell'intero addome e possiede una sensibilità e una specificità del $100 \%$ nell'ACD. La MR, anche se assai accurata, non viene solitamente utilizzata come metodica di prima scelta a causa della limitata disponibilità e dei costi: I'US rappresenta, pertanto, la modalità di imaging di prima istanza nella valutazione del paziente con ACD e per la ricerca di eventuali diverticoli anche nel paziente candidato alla PD. Essa fornisce informazioni preziose circa la localizzazione della malattia e l'eventuale interessamento della parete intestinale, nonché sulle manifestazioni extraparietali, come fistole e ascessi; inoltre, può essere eseguita ripetutamente senza alcuna limitazione (13). L'aspetto ecografico del colon dipende dal suo contenuto e dal grado di distensione. In condizioni normali, presenta un pattern austrato in scansione longitudinale (Fig. 3) e una forma rotondeggiante in sezione assiale. La maggioranza degli studi considera normale uno spessore parietale fino a $4 \mathrm{~mm}$, mentre il diametro del grosso intestino dipende dal suo stato di distensione o contrazione. Come per lo studio US di qualsiasi struttura anatomica, è possibile utilizzare il cambiamento di decubito del paziente per ottimizzare la visualizzazione della regione interessata. II color-Doppler permette di valutare l'eventuale stato di infiammazione nonché la morfologia vascolare, nel sospetto di una massa neoplastica. Lo studio dei tessuti molli pericolici viene eseguito per individuare l'eventuale presenza di linfonodi e/o infiltrati infiammatori e di raccolte fluide. Ecograficamente, i diverticoli appaiono come estroflessioni della parete del colon contenenti solitamente materiale ecogeno rappresentato da aria, feci o coproliti (Fig. 4). L'ipertrofia della tonaca muscolare, l'edema e l'infiammazione determinano un ispessimento segmentario che si evidenzia all'US come un'area di aspetto ipoecogeno. Tali formazioni sacculari si riscontrano più frequentemente nel colon discendente e nel sigma («diverticoli levoposti»), mentre assai più raramente sono localizzate nel colon ascendente e nel cieco («diverticoli destroposti»).

\section{Ecografia nel posizionamento del catetere peritoneale}

Una delle chiavi del successo della PD è rappresentata dal tempestivo e sicuro posizionamento del catetere nella cavità 


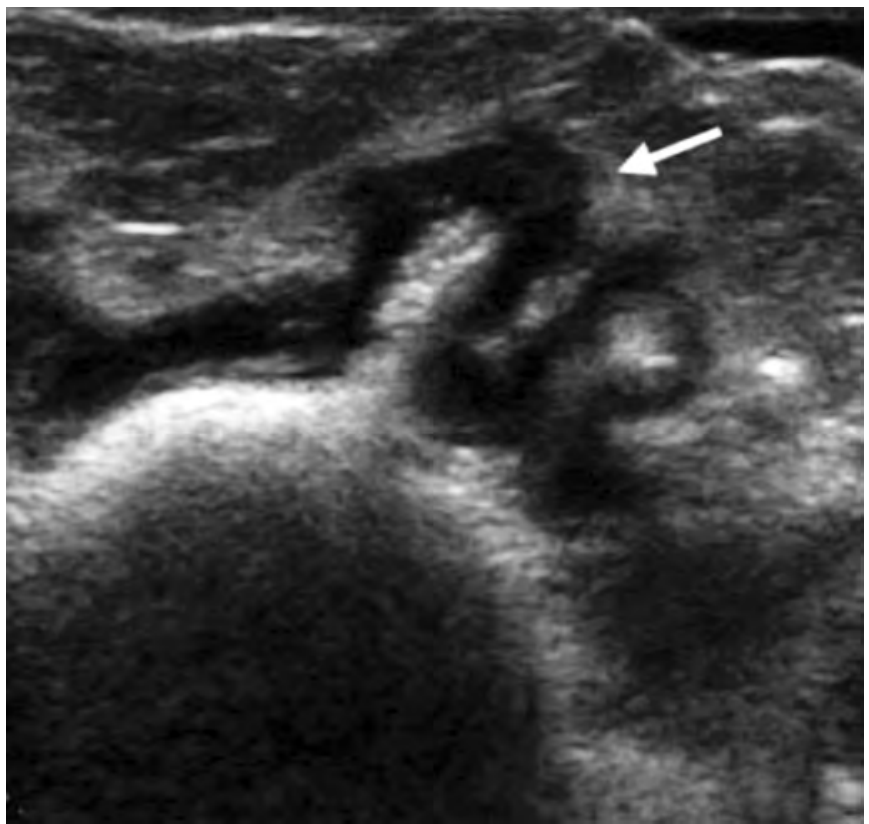

Fig. 4 - Diverticolo del colon con consensuale processo infiammatorio (freccia) del grasso pericolico.

peritoneale. Le tecniche più comunemente impiegate per l'inserimento del catetere peritoneale (PC) sono essenzialmente tre: la tecnica chirurgica, la via percutanea (tecnica di Seldinger ovvero con trocar) e, infine, la videolaparoscopia (VLS). Tuttavia, la metodica ideale rimane controversa. La tecnica chirurgica "open» costituisce l'approccio maggiormente utilizzato; la VLS, a sua volta, è l'unica in grado di consentire una visione diretta delle strutture intraperitoneali, il che potrebbe rappresentare il principale motivo del suo rapido espandersi (14-16). La via percutanea, per contro, rappresenta quella gravata dal minor numero di complicanze catetere-correlate e, pertanto, il suo utilizzo sarebbe auspicabile. Tale tecnica, inoltre, può essere resa maggiormente sicura dall'assistenza ecografica e fluoroscopica. L'utilizzo dell'US B-mode e del color-Doppler come guide all'introduzione dell'ago o del trocar permette di evitare le più comuni complicanze legate alla metodica, rappresentate soprattutto da perforazioni intestinali, puntura accidentale della vescica ed eventuali danni dei vasi epigastrici. Inoltre, essi possiedono altri indubbi vantaggi, che possono essere così schematizzati: 1) non è necessaria alcuna preparazione intestinale prima dell'intervento. Il paziente può introdurre liquidi per assumere i farmaci anche dopo la mezzanotte del giorno prima dell'intervento; 2) non è necessaria una prolungata degenza ospedaliera, dal momento che possono essere eseguiti in Day Surgery; 3) sono richiesti solo pochi dati di laboratorio, essenzialmente i parametri relativi alla coagulazione; 4) il paziente riceve solo una sedazione cosciente, evitando i potenziali rischi e complicanze dell'anestesia generale; 5) alla maggioranza dei pazienti vengono somministrate solo minime quantità di farmaci endovenosi durante la procedura ed essi possono riassu- mere le abituali terapie per via orale immediatamente dopo l'intervento. La descrizione della tecnica di posizionamento del PC esula, ovviamente, dagli scopi della presente trattazione, ma eccellenti lavori sull'argomento sono stati pubblicati, tra gli altri, da Abdel-Aal et al (17) e da Golay et al (18).

Le principali complicanze perioperatorie del posizionamento del PC sono rappresentate dalla lesione dell'arteria epigastrica con formazione di ematomi più o meno estesi e dalla perforazione intestinale con conseguente peritonite. L'inserimento ecoguidato del PC permette l'identificazione delle strutture della parete addominale e delle anse intestinali durante l'ingresso dell'ago introduttore (o del trocar), mentre il color-Doppler consente il riconoscimento dei vasi epigastrici. In tal modo, la punta dell'ago (nel caso della tecnica di Seldinger) può essere condotta fin nella cavità peritoneale, evitando accuratamente le strutture descritte. Una volta che l'ago è giunto in sede, l'iniezione di mezzo di contrasto iodato consente di verificare con la fluoroscopia il corretto posizionamento all'interno della cavità peritoneale, evidenziando le anse intestinali e gli organi pelvici. La più frequente complicanza tardiva è, invece, costituita dal leakage di fluido (dialisato o ascite), riportato in una percentuale di casi variabile dall' $8.6 \%$ al $24 \%$ (19). L'impiego del PC a doppia cuffia, il posizionamento della cuffia profonda nello spessore del muscolo retto addominale e la configurazione swan-neck del catetere sembrano ridurre l'incidenza di tale complicanza (20). Per quanto riguarda, poi, la sopravvivenza a 1 anno del catetere, quest'ultima sembra essere addirittura migliore $(90 \%)$ nel gruppo con posizionamento percutaneo rispetto al gruppo (82\%) con posizionamento per via chirurgica classica (21). Possiamo, pertanto, concludere affermando che I'uso della guida ecografica associata alla fluoroscopia rende la tecnica percutanea di posizionamento del PC sicura e poco invasiva; essa rappresenta una valida alternativa alla chirurgia classica o «blind».

\section{Ecografia nelle infezioni dell'exit-site e del tunnel sottocutaneo}

Le infezioni legate al PC rappresentano una causa frequente di drop-out dalla PD, predisponendo e, talora, accompagnando lo sviluppo di peritoniti. È importante, soprattutto dal punto di vista terapeutico, distinguere l'infezione dell'exit-site (ESI) da quella del tunnel sottocutaneo (TI), essendo quest'ultima più difficile da trattare $\mathrm{e}$, di conseguenza, esponendo maggiormente a rischi la sopravvivenza del catetere. La diagnosi di ESI e di TI è basata essenzialmente su criteri clinici. L'infezione dell'exit-site si manifesta soprattutto con arrossamento, dolenzia e dolorabilità e, talora, con la fuoriuscita di una secrezione sierosa, siero-ematica o purulenta dal punto di ingresso del PC. È importante valutare la presenza di croste, piccole secrezioni o sanguinamenti e bottoni di granulazione, poiché essi rappresentano segni di incompleta guarigione dell'exit-site, predisponendo, contemporaneamente, alla colonizzazione batterica. L'infezione del tunnel si sviluppa 

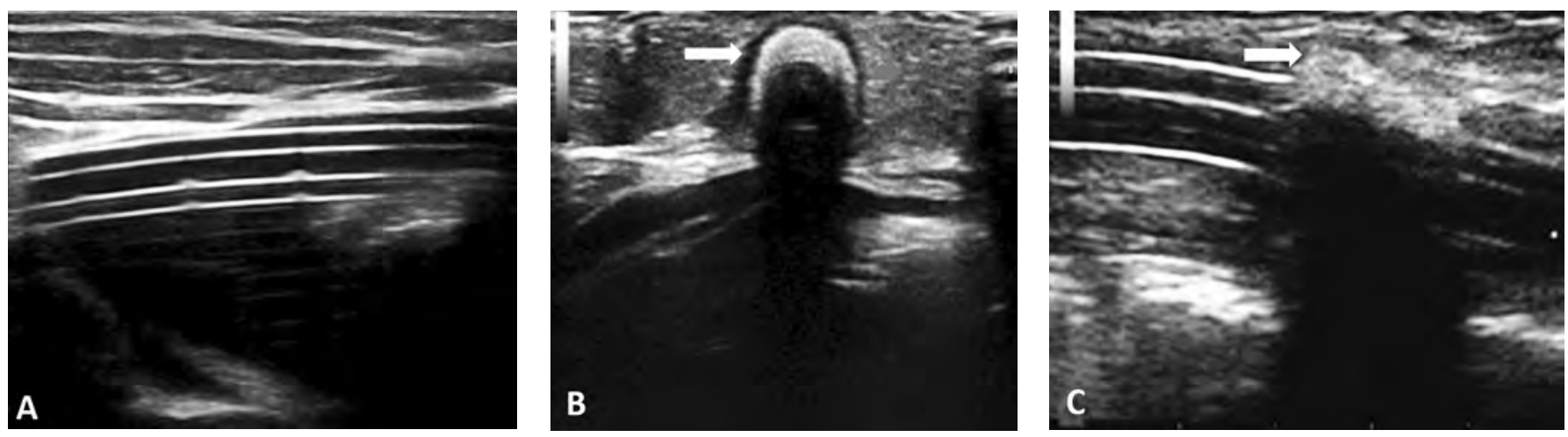

Fig. 5 - (A) Scansione longitudinale che mostra il caratteristico aspetto «a sandwich» del catetere di Tenckhoff. Scansione assiale (B) e longitudinale (C) che evidenziano la cuffia superficiale del catetere di Tenckhoff. La cuffia, localizzata nel tessuto sottocutaneo, appare iperecogena con caratteristica ombra acustica posteriore.

nel tragitto sottocutaneo del PC e può rendersi evidente con arrossamento e dolorabilità lungo il decorso del catetere, ma può comparire anche senza apparenti segni clinici. Può verificarsi una positività o meno dell'esame colturale. Solitamente, la TI compare in presenza di ESI. L'importanza dell'ecografia nella diagnosi precoce e nel trattamento delle TI nei pazienti in PD è ben documentata, laddove altre indicazioni risultano, invece, meno chiare. L'ecografia possiede un'eccellente sensibilità nella diagnosi di TI clinicamente occulte. Inoltre, I'US del tunnel è utile per valutare il tragitto sottocutaneo del PC in tutti i casi di ESI, nel follow-up di una TI precedentemente diagnosticata e nella valutazione di peritoniti recidivanti o resistenti alla terapia. Più controverso appare, invece, il ruolo dell'ecografia del tunnel in corso di peritoniti, febbri di origine sconosciuta o, semplicemente, come routine clinica (22). L'esame ecografico del tunnel sottocutaneo può essere eseguito con una sonda convex e/o lineare con frequenza variabile tra 5 e $18 \mathrm{MHz}$ e con il paziente in posizione supina. Attraverso scansioni longitudinali e assiali sarà possibile visualizzare il PC in tutta la sua lunghezza, comprendente il tratto sottocutaneo, il segmento intraddominale con i fori posti alla sua estremità, nonché il lume del catetere stesso. Sul piano sagittale, il PC appare come una struttura a "sandwich», formata da un banda iperecogena a doppio strato che ne rappresenta la parete anteriore e posteriore (Fig. 5A). In condizioni normali, è possibile visualizzare una rima ipoecogena esterna come conseguenza dell'ombra acustica posteriore prodotta dall'ecogenicità parietale. È, pertanto, opportuno che il PC venga esaminato secondo diverse angolazioni per poter distinguere l'artefatto da una raccolta fluida pericateterale. La cuffia superficiale e quella profonda del catetere appaiono ecograficamente come un menisco iperecogeno privo di un segnale acustico posteriormente (Fig. 5B, C). La semeiotica ecografica in caso di TI si caratterizza per la presenza di una raccolta fluida sottocutanea e per l'evidenza di un'iperemia pericateterale al color-Doppler. La raccolta fluida appare come un'area ipoecogena che circonda il PC e infiltra i tessuti adiacenti
(Fig. 6). Le dimensioni della raccolta potrebbero essere in grado di predire quali pazienti avranno una migliore risposta terapeutica, selezionando, eventualmente, quelli in cui la rimozione del catetere può rappresentare la scelta più corretta. La presenza di una zona iperecogena intorno alla cuffia superficiale delle dimensioni maggiori di un millimetro dopo un ciclo adeguato di terapia antibiotica e il contemporaneo interessamento di quella profonda sembrerebbero associati a una prognosi clinica peggiore; in caso di ESI da Pseudomonas aeruginosa l'outcome clinico è, comunque, sfavorevole, indipendentemente dai segni ecografici (23). Molte TI decorrono in maniera silente. II numero complessivo di casi diagnosticati, rispetto a quelli riconosciuti solo clinicamente, aumenta in maniera significativa con il ricorso all'indagine ecografica $(24,25)$. In conclusione, l'ecografia del tunnel assume notevole importanza nella diagnosi precoce di un suo eventuale coinvolgimento in corso di ESI, per il follow-up durante una terapia per TI e per una valutazione della prognosi, specialmente in caso di infezione profonda. Dal momento che, in corso di ESI causata da $S$. aureus, si verifica il frequente interessamento del tunnel, è consigliabile eseguirne l'esame ecografico in tutti questi casi. In caso di TI si raccomanda di ripetere I'US almeno ogni due settimane. Allo stesso modo, l'ecografia del tunnel dovrebbe essere effettuata in caso di peritonite con contemporanea ESI e in pazienti con peritonite ricorrente o persistente. Viceversa, non rappresentano indicazioni per l'esame: lo screening di routine, la ricerca di foci infettivi nei pazienti con elevati livelli proteina $\mathrm{C}$ reattiva o febbre e le peritoniti senza ESI e dolore lungo il decorso del catetere e senza altri segni di infezione dell'exit-site.

\section{Ecografia nella peritonite sclerosante incapsulante}

La peritonite sclerosante incapsulante (ESP) o peritonite sclerosante è un processo infiammatorio caratterizzato dalla progressiva deposizione di una spessa cotenna fibrosa sulla membrana peritoneale. Si manifesta in circa l' $1 \%$ dei 

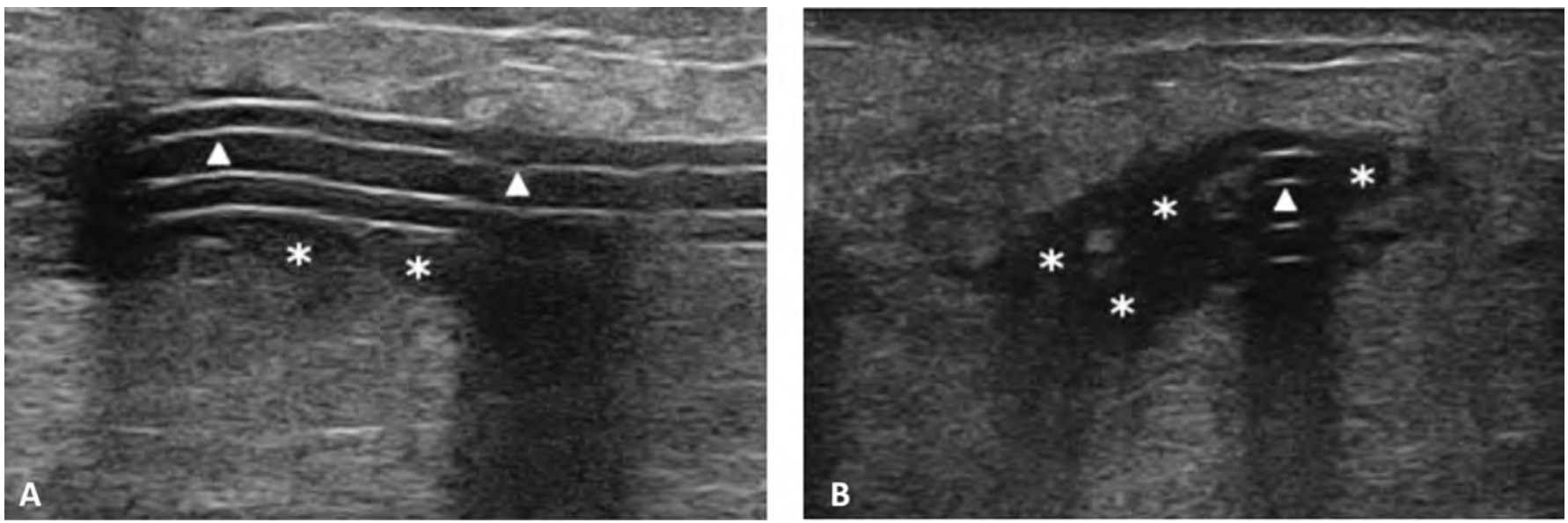

Fig. 6 - Ecografia del tunnel sottocutaneo in paziente con TI (vedi testo). Le frecce indicano il catetere di Tenckhoff in scansione longitudinale (A) e assiale (B). È, inoltre, ben evidente la zona ipo-anecogena circostante il catetere $\left({ }^{*}\right)$, espressione di raccolta fluida infiammatoria pericateterale.
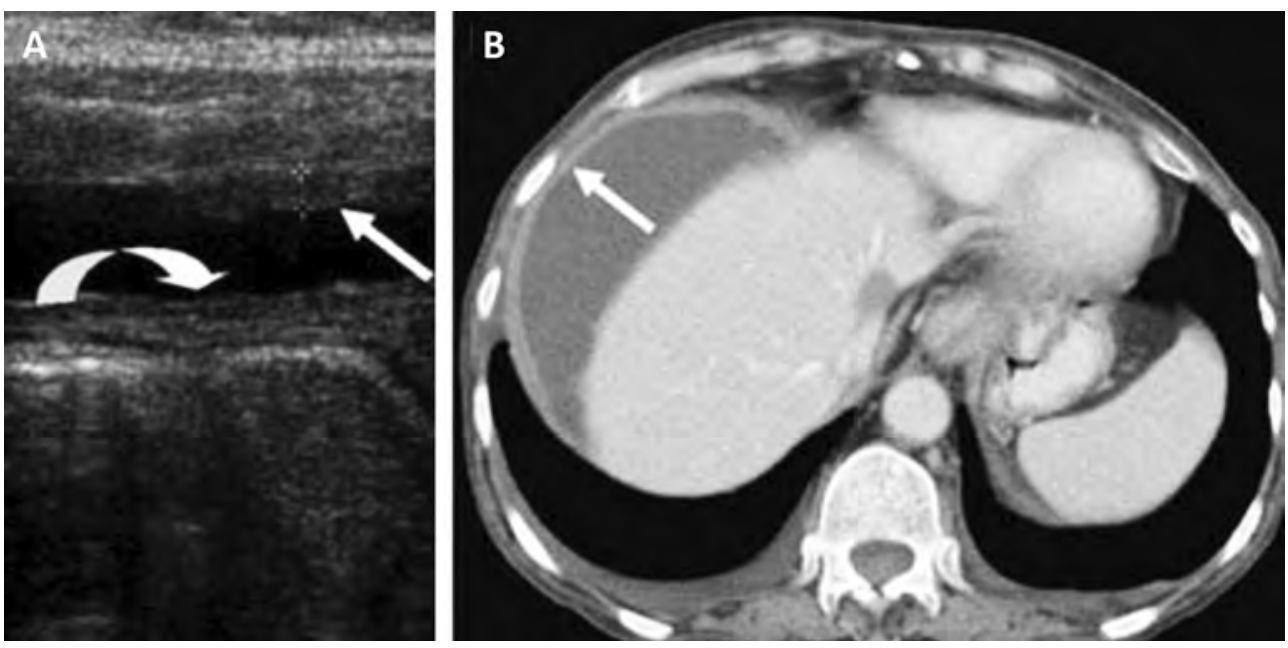

Fig. 7 - Ispessimento del peritoneo in corso di ESP (v. testo). (A) Ecografia: ispessimento del foglietto parietale (freccia dritta) e viscerale (freccia curva) della sierosa, tra loro separati da modeste quantità di fluido libero intaperitoneale. (B) CT con mdc in fase portale tardiva nello stesso paziente. Ispessimento del peritoneo parietale (freccia).

pazienti in PD con prevalenza progressivamente crescente con la durata del trattamento, potendo raggiungere il $20 \%$ a 8 anni (26). La sua eziologia è attualmente sconosciuta; sono noti, tuttavia, alcuni fattori, oltre alla già citata durata della PD, in grado di aumentarne il rischio di insorgenza, essenzialmente rappresentati dal tipo di soluzioni utilizzate e dagli episodi di peritonite, siano essi di natura chimica o batterica $(27,28)$. Il tempestivo riconoscimento della ESP riveste un'importanza clinica fondamentale, poiché la pronta interruzione della PD può prevenire la progressione e le ulteriori complicanze di questa patologia. Le alterazioni più precoci comprendono l'ispessimento peritoneale e la comparsa di calcificazioni della sierosa in presenza di segni clinici non specifici, come dolori addominali tipo colica o deficit di ultrafiltrazione. È altrettanto importante individuare il precoce coinvolgimento viscerale e parietale, poiché ciò potrebbe rappresentare la base del potenziale sviluppo di complicanze intestinali.

L'ecografia addominale eseguita in presenza del dialisato
( «con addome pieno») presenta una buona sensibilità per la diagnosi di ESP, anche se complessivamente minore rispetto a quella della $\mathrm{CT}$. L'addome viene esaminato con una sonda convex multifrequenza (2-5 MHz) e, successivamente, con un trasduttore lineare ad alta frequenza (6-10 MHz) per una valutazione del peritoneo. I segni ecografici caratteristici sono rappresentati da: 1) aumento della peristalsi nelle anse dilatate del piccolo intestino; 2) aderenza delle anse intestinali alla parete posteriore dell'addome; 3 ) ispessimento peritoneale, sia viscerale che parietale; 4) aree ipoecogene, corrispondenti a raccolte ascitiche loculate. È stata trovata una correlazione positiva tra la durata del trattamento con PD e lo spessore del peritoneo parietale; quest'ultimo può essere valutato in maniera semplice e non invasiva con l'ecografia (Fig. 7). Sebbene rappresenti un aspetto abbastanza frequente in corso di ESP, l'aumento dello spessore peritoneale non può essere considerato un segno specifico, potendo aumentare con l'età, il peso corporeo e l'altezza dell'individuo (29). Non è stato possibile individuare con certezza un va- 
lore tale di cut-off per lo spessore oltre il quale sia indicata I'interruzione della metodica: se il sospetto di ESP viene posto su tale base, potrebbe essere opportuno considerare l'eventualità di una biopsia del peritoneo (con tutti i rischi connessi). Le calcificazioni peritoneali, sia parietali che viscerali, possono essere presenti o mancare, divenendo via via più diffuse nel tempo. Possono essere osservate sia all'indagine US che all'esame diretto; tuttavia, la CT risulta assai più sensibile, particolarmente negli stadi precoci.

\section{Conclusioni}

Pur risentendo dei limiti legati alla dipendenza dall'operatore, l'indagine ecografica può essere utilmente impiegata nella maggior parte delle procedure e in numerose complicanze connesse al trattamento dialitico peritoneale. Essa offre il vantaggio di una prima diagnosi non invasiva in tempi rapidi, in ambulatorio o in corsia, indirizzando eventualmente verso tecniche di imaging più sofisticate o di secondo livello (CT, MR). Alcune indicazioni dell'ecografia sono ben codificate nel paziente in PD (p. es., infezioni dell'exit-site e del tunnel sottocutaneo), laddove altre risultano meno chiare (p. es., screening nella routine clinica e ricerca di foci infettivi senza segni di infezioni dell'exit-site o del tunnel sottocutaneo). II successo della tecnica è, tuttavia, strettamente dipendente dalla competenza dell'operatore e dalla sua familiarità con la metodica dialitica peritoneale.

\section{Disclosures}

Financial support: No financial support was received for this submission. Conflict of interest: The authors have no conflict of interest.

\section{Bibliografia}

1. Vandeman FN, Meilstrup JW, Nealey PA. Acoustic prism causing sonographic duplication artifact in the upper abdomen. Invest Radiol. 1990;25:658.

2. Yeh HC. Ultrasonography of peritoneal tumors. Radiology. 1979;133:419-24.

3. Derchi LE, Solbiati L, Rizzatto G, De Pra L. Normal anatomy and pathological changes of the small bowel mesentery: US appearance. Radiology. 1987;164:649-52.

4. Healy JC, Reznek RH. The peritoneum, mesenteries and omenta: normal anatomy and pathological processes. Eur Radiol. 1998;8:886-900.

5. Ferrari FS. In Peritoneo e Retroperitoneo. Collana Monotematica di Ecografia (diretta da M. Bazzocchi). Casa Editrice IdelsonGnocchi. 1997;(vol. 14):13.

6. Sigel B, Golub RM, Loiacono LA, et al. Technique of ultrasonic detection and mapping of abdominal wall adhesions. Surg Endosc. 1991;5:161-5.

7. Folvik G, Bjerke-Larssen T, Odegaard S, et al. Hydronography of the small intestine: comparison with radiologic barium study. Scand J Gastroenterol. 1999;34;176-82.

8. Fraquelli $M$, Colli $A$, Casazza $G$, et al. Role of sonography in detection of Crohn disease: meta-analysis. Radiology. 2005; 236: 95-101.
9. Drews $\mathrm{BH}$, Barth TF, Hänle MM, et al. Comparison of sonographically measured bowel wall vascularity, histology and disease activity in Crohn's disease. Eur Radiol. 2009;19:1379-86.

10. Neye H, Voderholzer W, Rickes S, et al. Evaluation of criteria for the activity of Crohn's disease by power Doppler sonography. Dig Dis. 2004;22:67-72.

11. Spalinger J, Patriquin $\mathrm{H}$, Miron MC, et al. Doppler US in patients with Crohn disease: vessel density in the diseased bowel reflects disease activity. Radiology. 2000;217:787-91.

12. Maconi G, Bollani S, Bianchi Porro G. Ultrasonography detection of intestinal complications in Crohn's disease. Dig Dis. Sci 1996;41:1643-8.

13. Rioux M, Gagnon J. Imaging modalities in the puzzling word of inflammatory bowel diasease. Abdom Imaging. 1997;22:173.

14. Ash SR. Chronic peritoneal dialysis catheters: effect of catheter design, material and location. Semin Dial. 1990;3:39-46.

15. Ogunc G, Tuncer M, Ogunc D, Yardimsever M, Ersoy F. Laparoscopic omental fixation technique vs open surgical placement of peritoneal dialysis catheters. Surg Endosc. 2003;17:1749-55.

16. Zaman F, Pervez A, Atray NK, et al. Fluoroscopy-assisted placement of peritoneal dialysis catheters by nephrologists. Semin Dial. 2005;18:247-51.

17. Asif A, Byers $P$, Vieira CF, Preston RA, Roth D. Diagnostic and interventional nephrology. Am J Therapeutics. 2002;9:530-6.

18. Abdel-Aal AK, Joshi KA, Seddekni S, Maya ID. Fluoroscopic and sonographic guidance to place peritoneal catheters: How we do it. Am J Roentgen. 2009;192:1085-9.

19. Golay V, Trivedi M, Roychowdhary A, et al. Ultrasoud-guided CAPD catheters insertion. Perit Dial Int. 2013;33(4):454-8.

20. Ozener C, Bihorac A, Akoglu E. Technical survival of CAPD catheters: comparison between percutaneous and conventional surgical placement techniques. Nephrol Dial Transplant. 2001;16: 1893-9.

21. Apostolidis NS, Panoussopoulos DG, Manouras AJ, Pararas BN, Voudiklari SG, Zirogiannis PN. The use of TWH catheters in CAPD patients: fourteen-year experience in technique survival and complication rates. Perit Dial Int. 1999;18:424-8.

22. Vychytil A, Lilaj $T$, Lorenz $M$, Hörl WH, Haagweber $M$. Ultrasonography of the catheter tunnel in Peritoneal Dialysis patients: What are the indications? Am J Kidney Dis. 1999;33(4):722-7.

23. Kwan TH, Ka-Hang-Tong M, Syu YP, et al. Ultrasonography in the management of exit-site infections in peritoneal dialysis patients. Nephrology. 2004;9(6):348-52.

24. Plum J, Sudkamp S, Grabensee B. Results of ultrasound-assisted diagnosis of tunnel infections in continuous ambulatory peritoneal dialysis. Am J Kidney Dis. 1994;23:99-104.

25. Vychytil A, Lorenz M, Schneider B, Hörl WH, Haagweber M. New criteria for management of catheter infections in peritoneal dialysis patients using ultrasonography. J Am Soc Nephrol. 1998;19:290-6.

26. Rigby RJ, Hawley CM. Sclerosing peritonitis: the experience in Australia. Nephrol Dial Transplant. 1998;13(1):154-9.

27. Ti JP, Al-Aradi A, Conlon PJ, Lee MJ, Morrin MM. Imaging features of encapsulating peritoneal sclerosis in continuous peritoneal dialysis patients. AJR Am J Roentgenol. 2010;195(1): W50-4.

28. Dobbie JW. Pathogenesis of peritoneal fibrosing syndromes (Sclerosing peritonitis) in peritoneal dialysis. Perit Dial Int. 1992;12(1):176-9.

29. Duman S, Ozbek SS, Gunay ES, et al. What does peritoneal thickness in peritoneal dialysis patients tell us? Adv Perit Dial. 2007;23:28-33. 